\title{
WhatsApp como ferramenta de trabalho do nutricionista
}

\author{
WhatsApp as a nutritionist job tool \\ WhatsApp como herramienta de trabajo del nutricionista
}

Carla Gabriele de Moura Silva ${ }^{1}$, Maria Clara Leal Cardoso ${ }^{1}$, Claudiane Batista de Sousa ${ }^{1}$, Tamires da Cunha Soares ${ }^{1}$, Rauene Raimunda de Sousa ${ }^{1}$, Kislley Almondes ${ }^{1}$, Ellaine Santana de Oliveira ${ }^{1}$, Gustavo Almondes Teixeira ${ }^{1}$, Victor Alves de Oliveira ${ }^{1}$, Sabrina Almondes Teixeira ${ }^{1 *}$.

\section{RESUMO}

Objetivo: Avaliar o perfil de utilização do WhatsApp na prática profissional do nutricionista. Métodos: Tratase de estudo transversal, descritivo, de caráter quantitativo e qualitativo, onde utilizou-se um questionário direcionados aos nutricionistas do município de Picos-PI. Foram incluídos aqueles profissionais que aceitaram participar da pesquisa, por meio da disponibilização de acesso aos seus respectivos locais de trabalho, e excluídos aqueles que não estavam com o cadastro no Conselho Regional do Nutricionista regularizado. Resultados: Observou-se que a utilização do aplicativo WhatsApp funciona como ferramenta útil de trabalho, mostrando-se eficaz, prático e acessível, além de melhorar a relação interpessoal com os grupos de trabalho envolvidos. Entretanto, a ocorrência de invasão de privacidade, assuntos paralelos e inconveniência foram os pontos negativos mais relatados. Conclusão: $O$ aplicativo mostrou-se eficaz, evidenciando assim alto grau de contribuição como ferramenta de trabalho do profissional nutricionista, porém trazendo para seu meio assuntos inconvenientes, portanto sendo necessário atenção no momento de seu uso.

Palavras-chave: Tecnologia, Nutricionista, Relações Interpessoais, Desempenho profissional.

\begin{abstract}
Objective: To evaluate the profile of the use of WhatsApp in the professional practice of the nutritionist. Methods: This was a descriptive, cross-sectional, quantitative and qualitative study, where a questionnaire was used for nutritionists in the city of Picos-PI. Those professionals who accepted to participate in the research were included, through the provision of access to their respective places of work and excluded those who were not registered with the Regional Nutritionist Council regularized. Results: It was observed that the use of the WhatsApp application works as a useful work tool, proving to be effective, practical and accessible, as well as improving the interpersonal relationship with the working groups involved. However, the occurrence of invasion of privacy, side issues and inconvenience were the most commonly reported negatives. Conclusion: The application showed to be effective, thus evidencing a high degree of contribution as a tool of work of the professional nutritionist, however bringing to his environment inconvenient subjects, therefore being necessary attention at the moment of its use.
\end{abstract}

Key words: Technology, Nutricionists, Interpersonal Relations, Work Performance.

\section{RESUMEN}

Objetivo: Evaluar el perfil de utilización de WhatsApp en la práctica profesional del nutricionista. Métodos: Se trata de un estudio transversal, descriptivo, de carácter cuantitativo y cualitativo, donde se utilizó un cuestionario dirigido a los nutricionistas del municipio de Picos-PI. Se incluyeron aquellos profesionales que aceptaron participar de la investigación, a través de la disponibilidad de acceso a sus respectivos lugares de trabajo, y excluidos aquellos que no estaban con el registro en el Consejo Regional del Nutricionista regularizado. Resultados: Se observó que el uso de la aplicación WhatsApp funciona como herramienta útil

${ }^{1}$ Universidade Federal do Piauí (UFPI), Picos-Piauí. *E-mail: sabrina.almondes@hotmail.com

2 Faculdade Raimundo de Sá (FRSá), Picos-Piauí.

SUBMETIDO EM: 1/2019

ACEITO EM: 2/2019

PUBLICADO EM: 7/2019

REAS/EJCH | Vol. Sup.26 | e338 | DOI: https://doi.org/10.25248/reas.e338.2019 Página 1 de $\mathbf{9}$ 
de trabajo, mostrándose eficaz, práctico y accesible, además de mejorar la relación interpersonal con los grupos de trabajo involucrados. Sin embargo, la ocurrencia de invasión de privacidad, asuntos paralelos e inconveniencia fueron los puntos negativos más relatados. Conclusión: La aplicación se mostró eficaz, evidenciando así alto grado de contribución como herramienta de trabajo del profesional nutricionista, pero trayendo para su medio asuntos inconvenientes, por lo tanto siendo necesaria atención en el momento de su uso.

Palabras clave: Tecnología, Nutricionistas, Relaciones Interpersonales, Rendimiento Laboral.

\section{INTRODUÇÃO}

O nutricionista é um profissional de saúde generalista, responsável pela promoção da segurança alimentar e atenção nutricional em todas as áreas do conhecimento e atuação em que a alimentação e a nutrição sejam fundamentais para contribuir com a promoção, a manutenção e a recuperação da saúde e para a prevenção de doenças de indivíduos ou coletividades, através de recomendações adequadas e equilibradas de nutrientes, contribuindo para a melhoria da qualidade de vida, com princípios éticos e morais, respeitando a realidade econômica, social e cultural (RECINE E et al., 2012).

As ferramentas utilizadas pelo nutricionista são de suma importância para alcance e verificação de indicadores diretos, tendo como exemplo, os dados antropométricos, tais como circunferências, pregas cutâneas, peso e suas relações com altura e idade, utilizando utensílios como balança, fita métrica, adipômetro, estadiômetro (CFN, 2008).

Outras ferramentas que podem vir a serem utilizadas pelos profissionais são os softwares, como por exemplo os que ajudam nos cálculos dietéticos, softwares de auxílio a dieta como aplicativos que acionam lembretes para ingestão diária de água (QUADROS MRR; DIAS JS; MORO CMC, 2004).

Aplicativos também podem ser intitulados como software, por exemplo o WhatsApp, que é descrito como um software multiplataforma de mensagens súbitas e chamadas de voz para smartphones, que além de mensagens de texto, os usuários podem enviar fotos, vídeos, áudios de mídia e, na nova atualização, podem realizar chamada de voz e chamada de vídeo para qualquer contato de sua agenda que possua WhatsApp, constituindo então uma nova interface entre real e virtual (ROCHA D et al., 2017).

Como ponto positivo, em relação ao aplicativo, apresenta-se a praticidade como papel principal e fundamental, se tornando um meio viável de apoio ao nutricionista, possibilitando a interação imediata entre profissional e paciente/cliente, promovendo seu trabalho, aumentando suas vendas, dissipando seu marketing e amplificando informações dos seus serviços, podendo assim, influenciar o público alvo, também evidenciando pontos negativos como invasão de privacidade, assuntos paralelos e desvio de atenção, com isso o usuário deve atentar-se no momento da sua utilização (SOUZA JLA et al., 2015).

Como a busca pela utilização de novas tecnologias cresce gradualmente no mercado de trabalho, nessa perspectiva ferramentas práticas e de fácil acesso, como o WhatsApp, vêm ganhando espaço, com isso o objetivo desse estudo foi avaliar o perfil de utilização do WhatsApp na prática profissional do nutricionista.

\section{MÉTODOS}

Trata-se de um estudo transversal, descritivo, de caráter quantitativo e qualitativo, o qual procedeu-se na cidade de Picos-PI, em diversos locais de trabalho dos profissionais nutricionistas, considerando as diversas áreas de atuação. Na definição da amostra em estudo levou em consideração a totalidade dos nutricionistas atuantes na cidade em questão, onde foi realizado previamente o quantitativo destes, assim como os respectivos locais de trabalho. Para isso pesquisamos informações disponíveis junto ao $\mathrm{CRN}-6$, secretarias de educação municipal e estadual, secretarias de saúde municipal e estadual e instituições de ensino superior. Neste sentido, foram incluídos nutricionistas que aceitaram participar da pesquisa, por meio da disponibilização de acesso aos seus respectivos locais de trabalho, e excluídos aqueles que não estavam 
com o cadastro no CRN (Conselho Regional do Nutricionista) regularizado, chegando a um total de 50 profissionais. Como instrumento para coleta de dados foi utilizado um questionário, desenvolvido com base no estudo de Teixeira GA (2017), com adaptações direcionadas as atividades profissionais do nutricionista. Este é composto por 15 questões, objetivas e subjetivas, sendo as sete primeiras voltadas ao perfil socioeconômico e as demais voltadas aos aspectos específicos do estudo realizado, o qual circunda sobre o uso do WhatsApp como ferramenta de trabalho do nutricionista com suas respectivas vantagens e desvantagens.

Este projeto foi submetido e aprovado pelo Comitê de Ética em Pesquisa (CEP) da Universidade Federal do Piauí (UFPI) com CAAE oㅡ 86278518.5.0000.5214, assim cumprindo com as exigências da Resolução no 466/2012, do Conselho Nacional da Saúde (CFN, 2006a). Desta forma, após assinatura do Termo de Consentimento Livre e Esclarecido (TCLE), e consequentemente à consolidação da participação dos nutricionistas, foi realizada a aplicação do respectivo questionário.

Ressalta-se que o profissional e/ou seu respectivo secretário, foram contatados previamente para verificação da disponibilidade de participar da pesquisa e consequentemente agendar uma data e horário para se proceder a coleta de dados. No momento de coleta foi preservado a individualidade do profissional, sendo aplicado o questionário um a um e em ambiente cômodo e confortável. Após a coleta, os dados quantitativos foram tabulados e analisados com o auxílio do software Microsoft Excel 2013, obtendo dados percentuais os quais foram demonstrados posteriormente em gráficos e tabelas. A análise dos dados qualitativos foi realizada por meio de tendências de respostas, estas foram organizadas pela frequência de citações entre os participantes da pesquisa.

\section{RESULTADOS}

Os dados abaixo descritos fazem referência aos resultados obtidos após a aplicação dos questionários estruturados, destinados a nutricionistas atuantes na cidade de Picos-PI.

Tabela 1 - Perfil socioeconômico dos nutricionistas atuantes em Picos-PI. $(n=50)$.

\begin{tabular}{ll}
\hline SEXO & \\
\hline Masculino & $10 \%$ \\
Feminino & $90 \%$ \\
\hline IDADE (ANOS) & $76 \%$ \\
\hline $20-30$ & $24 \%$ \\
$31-40$ & $0 \%$ \\
$>41$ & $28 \%$ \\
\hline RENDA MÉDIA MENSAL (SALÁRIO MINIMO) & $42 \%$ \\
\hline $1-2$ & $18 \%$ \\
$3-4$ & $12 \%$ \\
$5-6$ & \\
$>6$ & $38 \%$ \\
\hline VíNCULO PROFISSIONAL & $66 \%$ \\
\hline Autônomo & \\
Empregado & $14 \%$ \\
\hline TEMPO DE ATUAÇÃO (ANOS) & $32 \%$ \\
\hline$<1$ & $32 \%$ \\
$1-3$ & $16 \%$ \\
$4-6$ & $6 \%$ \\
$7-9$ & \\
$\geq 10$ & $12 \%$ \\
\hline GRAU DE FORMAÇÃO & $66 \%$ \\
\hline Superior completo & $20 \%$ \\
Especialização & $2 \%$ \\
Mestrado &
\end{tabular}

Fonte: Dados da Pesquisa, 2018. 
A Tabela 1 traz a descrição do perfil socioeconômico da população amostral, esta foi composta por 50 indivíduos de ambos os sexos, no entanto a maioria dos participantes era representado pelo sexo feminino (90\%). Quanto à faixa etária, predominaram-se indivíduos adultos jovens, sendo a faixa de 20 a 30 anos, a mais prevalente com $76 \%$ da amostra.

A renda média mensal foi outra variável avaliada, observando uma renda mais representativa para valores de 3 a 4 salários mínimos, no entanto houve, em menor proporção, valores superiores a 6 salários (12\%).

Quanto ao tipo de vínculo profissional a maior parte dos nutricionistas relataram ser empregados (66\%), seja por instituição públicas ou privadas, ressaltando que a maioria desses profissionais possuíam com pouco tempo de atuação, variando de 1 a 6 anos.

No que se diz respeito à qualificação, a maioria possuíam pelo menos especialização (66\%), seguido de mestrado (20\%) (Tabela 1).

A pesquisa abordou dez áreas de atuação, sendo a nutrição clínica a mais prevalente, com $56 \%$, seguida da alimentação coletiva, tanto em UAN como escolar, ambas com $26 \%$ (Gráfico 1). Das áreas de atuação avaliadas a Nutrição e Marketing foi a única que não esteve contemplada.

Gráfico 1 - Áreas de atuação dos nutricionistas que exercem atividade profissional em Picos-PI. 2018.

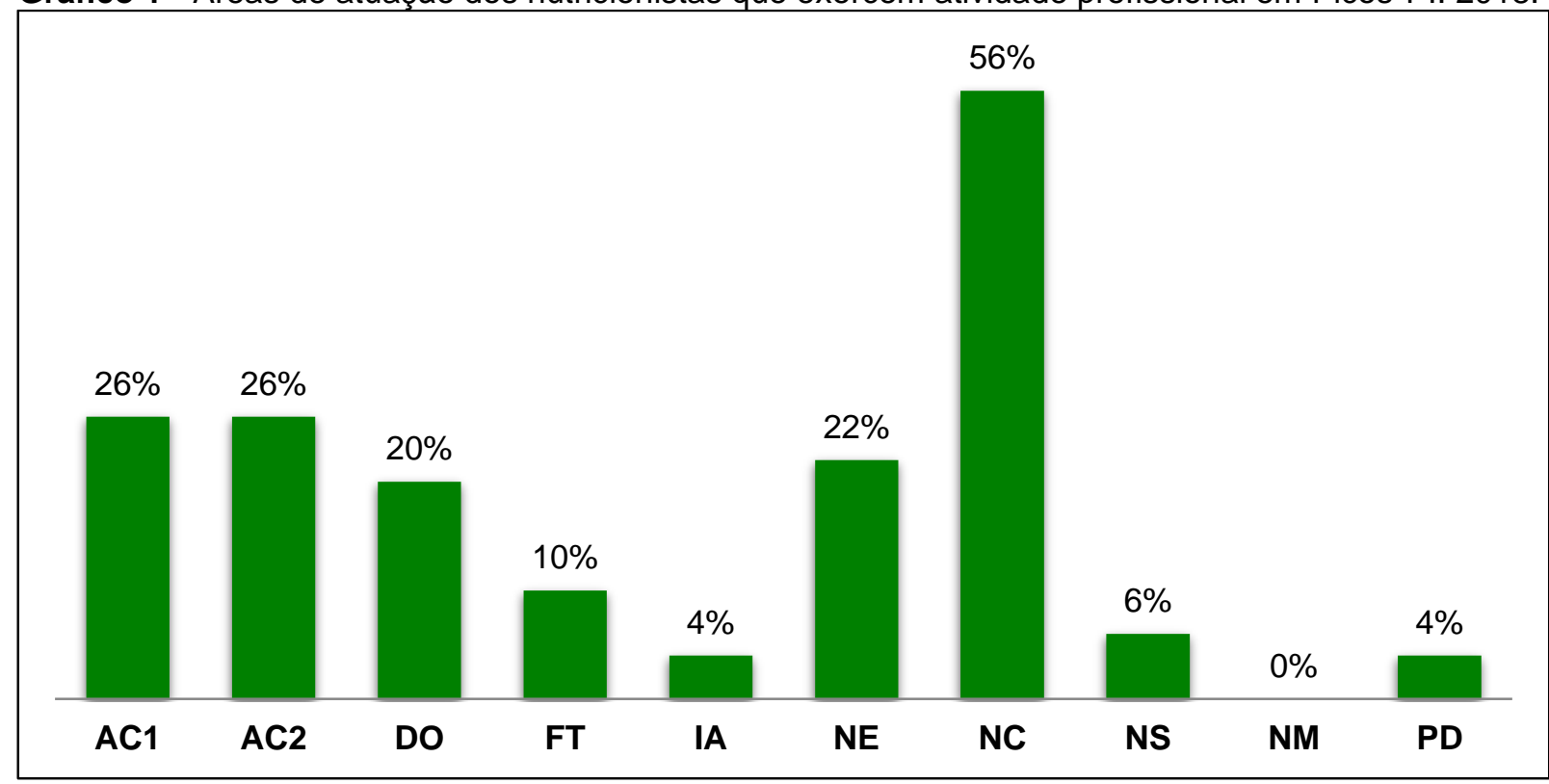

Fonte: Dados da Pesquisa, 2018.

*AC1: Alimentação coletiva (Unidade de Alimentação e Nutrição); AC2: Alimentação coletiva (Escolar); DO: Docência; FT: Fitoterapia; IA: Industria de Alimentos; NE: Nutrição esportiva; NC: Nutrição clínica; NS: Nutrição Social; NM: Nutrição e Marketing; PD: Personal Diet.

$\mathrm{Na}$ análise específica do WhatsApp (Tabela 2) observou-se unanimidade para o uso do aplicativo, tanto no âmbito pessoal como profissional, com período de utilização superior a 24 meses $(88 \%$ e $44 \%$, respectivamente).

$\mathrm{Na}$ avaliação da utilidade do aplicativo para prática profissional, 100\% dos nutricionistas relataram concordância, além de evidenciar um representativo grau de contribuição "alto" entre as respostas obtidas $(60 \%)$.

Vale ainda destacar que a formação/participação em grupos no WhatsApp, direcionados para a atuação do nutricionista foi uma prática notável, $68 \%$ de respostas positivas. 
Tabela 2 - Perfil de uso e de contribuição do WhatsApp, como ferramenta de trabalho, na prática profissional do nutricionista. 2018.

\begin{tabular}{|c|c|}
\hline \multicolumn{2}{|c|}{ USUÁRIO DO WHATSAPP } \\
\hline $\begin{array}{l}\text { Não } \\
\text { Sim }\end{array}$ & $\begin{array}{c}0 \% \\
100 \%\end{array}$ \\
\hline \multicolumn{2}{|c|}{ TEMPO DE USO PESSOAL DO WHATSAPP (MESES) } \\
\hline $\begin{array}{l}<6 \\
6-12 \\
12-24 \\
>24\end{array}$ & \begin{tabular}{|l|}
$2 \%$ \\
$2 \%$ \\
$8 \%$ \\
$88 \%$
\end{tabular} \\
\hline \multicolumn{2}{|c|}{ TEMPO DE USO PROFISSIONAL DO WHATSAPP (MESES) } \\
\hline $\begin{array}{l}<6 \\
6-12 \\
12-24 \\
>24\end{array}$ & $\begin{array}{c}4 \% \\
20 \% \\
32 \% \\
44 \%\end{array}$ \\
\hline \multicolumn{2}{|c|}{ WHATSAPP FERRAMENTA PROFISSIONAL UTIL } \\
\hline $\begin{array}{l}\text { Sim } \\
\text { Não }\end{array}$ & $\begin{array}{c}100 \% \\
0 \%\end{array}$ \\
\hline \multicolumn{2}{|c|}{ GRUPO PROFISSIONAL NO WHATSAPP } \\
\hline $\begin{array}{l}\text { Sim } \\
\text { Não }\end{array}$ & $\begin{array}{l}68 \% \\
32 \%\end{array}$ \\
\hline \multicolumn{2}{|c|}{ GRAU DE CONTRIBUIÇÃO PARA PROFISSÃO } \\
\hline $\begin{array}{l}\text { Muito alto } \\
\text { Alto } \\
\text { Médio } \\
\text { Baixo } \\
\text { Muito baixo }\end{array}$ & $\begin{array}{l}18 \% \\
60 \% \\
18 \% \\
4 \% \\
0 \% \\
\end{array}$ \\
\hline
\end{tabular}

Fonte: Dados da Pesquisa, 2018.

Também foi objeto deste estudo a avaliação qualitativa dos pontos positivos e negativos, ao que se refere à aplicação do WhatsApp na prática profissional do nutricionista. Pode ser observado no Gráfico 2 a frequência dos atributos positivos relacionados ao uso do aplicativo pelos nutricionistas, sendo a "agilidade", "facilidade na troca de informações" e "interação entre profissional e cliente" os pontos mais recorrentes, com $50 \%, 50 \%$ e $40 \%$, respectivamente.

Gráfico 2 - Pontos positivos relacionados ao uso do WhatsApp na pratica profissional dos Nutricionistas de Picos-PI.

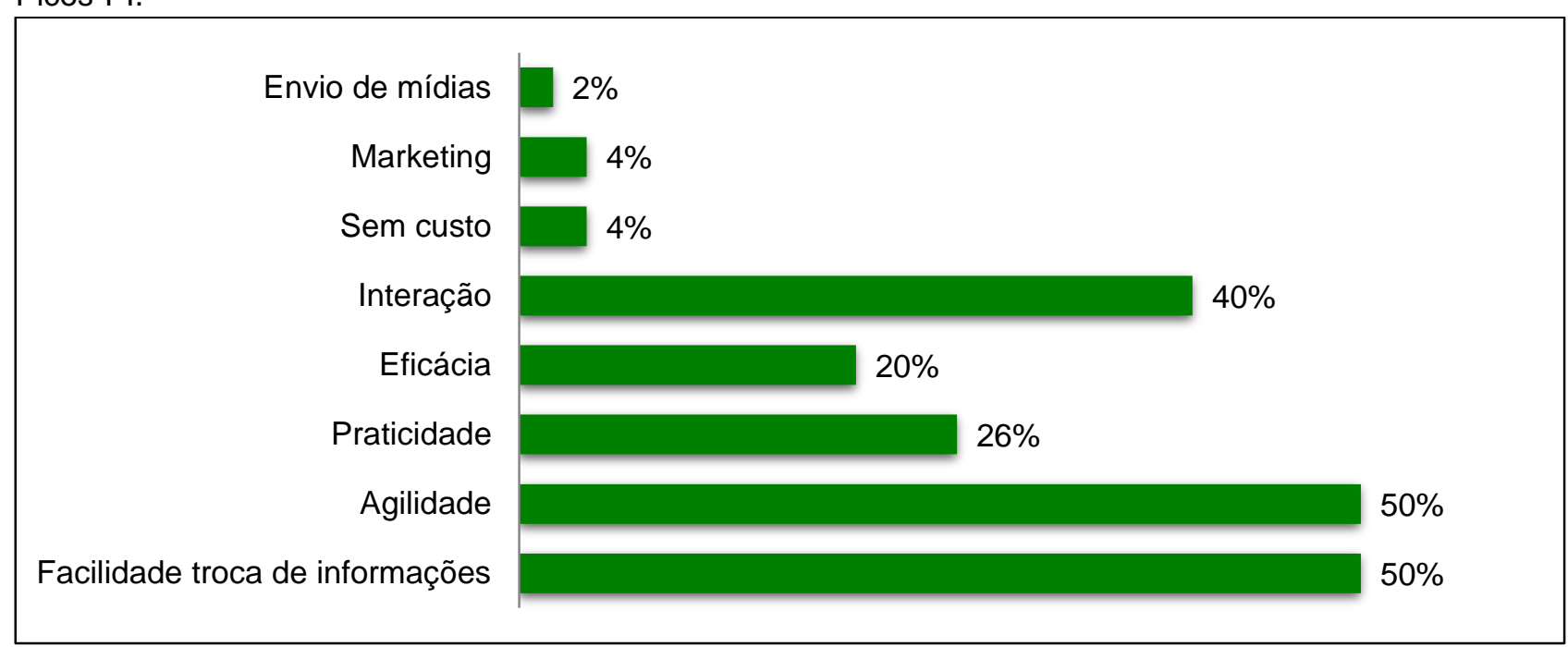

Fonte: Dados da Pesquisa, 2018. 
Os pontos negativos evidenciados estão descritos no Gráfico 3. Por meio deste podemos observar uma maior frequência de relatos para a "invasão de privacidade" (26\%), "assuntos paralelos" (20\%) e "inconveniência" (20\%).

Gráfico 3 - Pontos negativos relacionados ao uso do WhatsApp na pratica profissional dos Nutricionistas de Picos-PI. 2018.

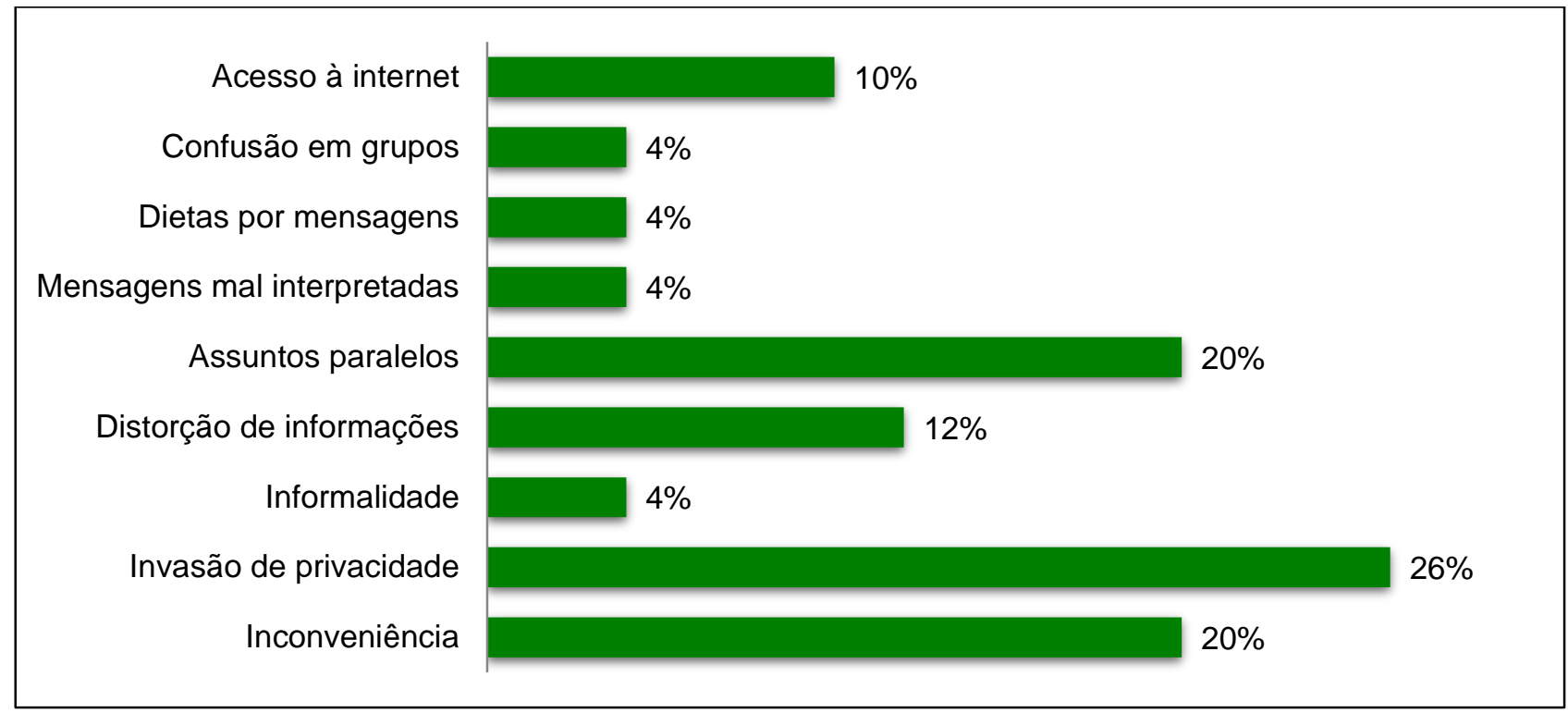

Fonte: Dados da Pesquisa, 2018.

\section{DISCUSSÃO}

Tendo em vista a análise dos resultados encontrados, observa-se uma predominância notável do público feminino (90\%), entre os Nutricionistas atuantes na cidade de Picos-PI. De acordo com Akutsu RC (2008), o curso de nutrição pode ser visto como uma profissão feminina apontando um percentual de $96,9 \%$, dados este de acordo com uma pesquisa realizada pelo CFN (2006b), o qual entrevistaram 2.492 nutricionistas, estratificados proporcionalmente por Regional (CRN), constatando que no Brasil as mulheres tem $96,5 \%$ de atuação comparado com o público masculino.

Quanto à faixa etária, mais de um terço da população (76\%) apresentam idade entre 20 e 30 anos. O CFN (2006b) também enfatiza em pesquisa que $79,4 \%$ dos Nutricionistas atuantes no Brasil possuíam idade entre 20 a 40 anos, mostrando semelhança aos resultados obtidos nesta pesquisa. Os resultados obtidos por Mello AL et al. (2012), também corroboram com este trabalho, no seu estudo, o qual visava caracterizar o perfil do nutricionista atuante no Programa de Alimentação e Nutrição do Escolar no Brasil, observou-se uma considerável representação para a faixa etária entre 20 e 30 anos (42,7\%).

Soar C e Silva CAM (2017), por meio do estudo que avaliou a histórico profissional de nutricionistas egressos de uma respectiva universidade, observaram que a faixa de renda mais prevalente teve variação entre 2 e 3 salários mínimos, valores estes que podem estar envolvidos com inúmeras variáveis, como por exemplo o local ou município que o mesmo atua, podendo ter um nível inferior aos outros profissionais do setor da saúde. Embora o resultado encontrado por esta pesquisa tenha evidenciado renda mais prevalente entre de 3 a 4 salários, vale ressaltar que esses valores encontrados ocorrem muitas vezes, pelos profissionais atuarem em mais de uma área, e consequentemente acumularem mais de um cargo.

Uma proposição que pode estar relacionada com resultados evidenciados para a renda é quanto ao vínculo empregatício. Neste estudo observou-se que a maioria dos entrevistados afirmaram ter mais de um vínculo empregatício, podendo este ser autônomo ou empregado. 
Segundo pesquisa feita por Rodrigues MK et al. (2007), que abordou as condições e perfil de trabalho dos nutricionistas em suas diferentes áreas de atuação, as respostas foram semelhantes à pesquisa discorrida nesse atual estudo. Vale salientar que dentre os nutricionistas avaliados nesta pesquisa, a maioria relatava possuir um segundo emprego por motivo de melhoria salarial e não apenas por sua realização profissional.

Acerca das afirmações supracitadas, apesar de observar neste estudo uma maior tendência para o vínculo empregado, pode-se concluir que as evidências relacionadas à forma de trabalho autônoma por nutricionistas, pode se relacionar à falta de oportunidade no mercado de trabalho ao longo dos últimos anos, colocando em questão um meio de sobrevivência, assim como a possibilidade de melhores remunerações.

Segundo Colonetti T et al. (2015), o curso de nutrição no Brasil surgiu há 70 anos, sendo que entre os anos de 1997 a agosto de 2009, houve grande expansão do número de cursos e, consequentemente, número de profissionais nutricionistas do país. Na cidade de estudo, Picos-PI, a profissão mencionada é nova no mercado de trabalho, estes tendo sua formação em diversas instituições, mas em especial na universidade instalada na própria cidade. $O$ curso de nutrição encontra-se presente há 11 anos na cidade, tendo sua primeira turma graduada há 6 anos. Estes dados justificam as evidencias quanto ao tempo de atuação dos profissionais deste estudo, apontando períodos entre 1 e 6 anos.

A qualificação profissional foi uma variável de interesse neste estudo, onde as mais relatadas foram especialização e mestrado, demonstrando semelhança aos estudos de Souza JCN et al. (2018), estes realizaram uma análise quanto aos egressos de um curso de graduação da região nordeste, encontrando $72,2 \%$ de egressos inseridos em especializações, $26,2 \%$ em mestrado e $6,4 \%$ em doutorado. É importante destacar que o fato dos nutricionistas desejarem se qualificar torna possível uma atuação mais qualificada, consequentemente agregando mais segurança e reconhecimento financeiro.

$\mathrm{Na}$ presente pesquisa abordou-se as dez áreas de atuação do profissional nutricionista, seguindo a classificação do CFN, verificando um forte destaque para a nutrição clínica, fato esse que pode ser justificado por ser uma especialidade de maior autonomia para o público recém-formado, já que a cidade onde os mesmos trabalham é um polo de saúde da macrorregião impulsionando assim a prática clínica. A área de alimentação coletiva (UAN e escolar) também foi bastante representada, podendo ser considerada uma área bastante promissora, principalmente devidos a obrigatoriedade legal do responsável técnico.

O estudo de Aguiar OB e Silva TM (2017), corroboram com os achados desta pesquisa, relatando maior prevalência para as áreas da nutrição clínica $(27 \%)$ e alimentação coletiva $(28,9 \%)$ em comparação com as demais áreas. Relato este também citado pelo CFN (2006b), os quais trazem dados nacionais de $40 \%$ de atuação na área de nutrição clínica e 32,2\% na alimentação coletiva.

Criado em 2009, mas somente em 2014, o WhatsApp tornou-se uns dos aplicativos mais utilizados no mundo (mais de 250 milhões de usuários), possibilitando o envio de mensagens instantâneas, com maior velocidade, menos custo e assim, diminuindo distâncias entre seus usuários (SOUZA AG et al., 2014). Como já mencionado, utilizado para a troca rápida e fácil de mensagens, envio de fotos, vídeos, documentos, e mídias em geral, o WhatsApp também é usado no âmbito profissional de diversas áreas.

Furlanetto GS (2017), apresenta o WhatsApp como ferramenta útil, a qual amplia contatos e atividades com informações e situações de trabalho, estimulando assim a participação independente de tempo e espaço. Dessa forma, para o autor esta ferramenta é de suma importância para a inovação de processos comunicativos interpessoais, facilitando com isso atividades profissionais. Este aplicativo tem permitido que pessoas e profissionais de diversas áreas fiquem mais próximos do público alvo, desta forma no presente estudo encontrou-se respostas onde o aplicativo WhatsApp é dito como ferramenta de utilidade para a prática profissional do nutricionista corroborando assim com o pensamento do autor.

Ficou evidente que todos os profissionais em estudo faziam uso rotineiro do aplicativo WhatsApp, fato que se associa com a afirmativa citada por Souza JLA et al. (2015), os quais dizem que o uso dos aplicativos móveis está cada vez mais presente no cotidiano das pessoas sendo interessante destacar a frequência do uso diário do mesmo. 
Quanto ao tempo de uso verificou período superior a 24 meses, o que vai de encontro com Souza AG et al (2014), onde relata que o aplicativo surgiu no Brasil em 2014 sendo assim de utilização recente.

Quanto ao uso do WhatsApp no âmbito profissional, observou-se representação significativa a partir dos últimos seis meses, no entanto a maior parte dos nutricionistas já utilizam este aplicativo a mais de 24 meses (44\%).

Vale ressaltar que a maior parte dos participantes desta pesquisa relatou representativa contribuição do aplicativo no contexto profissional (60\%). Relatos do estudo de Raiman L et al. (2017), condizem com estes achados. Os mesmos realizaram estudo sobre o uso do aplicativo WhatsApp no ambiente de trabalho da saúde, demonstrando eficácia como ferramenta de comunicação, visto apresentar alto grau de contribuição na interação interpessoal em grupo, permitindo assim maior conectividade entre os membros da equipe a qualquer momento durante o serviço, destacando assim a grande a aplicabilidade no ambiente clínico.

Na prática da docência, o aplicativo também é muito utilizado, como mostra o estudo de Alencar GA et al. (2015). Este buscou avaliar a forma como o WhatsApp pode auxiliar no ensino e aprendizagem das aulas de um curso superior presencial, encontrando inúmeras facilidades obtidas mediante da utilização do WhatsApp pelo professor, vindo a ser um grande aliado principalmente quando se trata de educação à distância, sendo o aplicativo palco de debates na construção de atividades, compartilhando informações e esclarecendo dúvidas, tanto por parte dos professores, da instituição ou do aluno.

Ademais, o celular se transformou em um aliado para o professor, e consequentemente os aplicativos, sendo utilizado como ferramenta de suporte à aula, auxiliando no repasse de informações sobre matérias, aulas, na tirada de dúvidas sobre os conteúdos, trabalhos e por fim argumentam que alunos mais tímidos ou que não venha a conseguir falar em público ou em sala de aula, tenham a oportunidade de se comunicar melhor com a utilização do aplicativo.

A avaliação qualitativa deste estudo evidenciou resultados tanto positivos como negativos relacionados ao uso do WhatsApp na prática profissional do nutricionista. Dentre os pontos positivos relatados com maior frequência destaca-se a agilidade, facilidade na troca de informações e interação interpessoal, já quantos aos negativos, a invasão de privacidade, assuntos paralelos e inconveniência foram os mais recorrentes.

Quanto esta abordagem Oliveira EDS et al. (2014), dá ênfase que para a eficácia na utilização deste aplicativo, é indispensável o planejamento e organização do mesmo devido a sua forma dinâmica e rápida na troca de mensagens, podendo assim vir a gerar problemas, resultando em negativação, como por exemplo obtidos por esse estudo a invasão de privacidade, assuntos paralelos e inconveniência, sendo assim, necessário cautela no momento do seu uso.

Pereira PC et al. (2015), complementa ao destacar a importância do dinamismo que esse aplicativo oferece, permitindo uma interação significativa entre os usuários, além da permissão que o mesmo oferece na transferência de grandes volumes de dados, podendo ser textos, imagens, áudios, mostrando assim a agilidade e praticidade que o WhatsApp pode proporcionar.

\section{CONCLUSÃO}

Se pôde constatar neste estudo que a utilização do aplicativo WhatsApp na prática profissional do nutricionista mostrou-se eficaz evidenciando assim alto grau de contribuição como ferramenta útil de trabalho. O aplicativo se torna de suma importância nos dias atuais pela sua agilidade nas trocas de informações e praticidade facilitando assim as atividades profissional dos nutricionistas com isso melhorando a comunicação interpessoal e o relacionamento com os grupos envolvidos, porém trazendo para seu meio assuntos paralelos e inconveniência, portanto sendo necessário atenção no momento do seu uso. Com isso a presente pesquisa vem como forma de contribuir e incentivar novos estudos nessa área, uma vez que, ajudam a aperfeiçoar os conhecimentos sobre o WhatsApp como ferramenta de trabalho do profissional nutricionista nas suas diversas áreas de atuação. 


\section{REFERÊNCIAS}

1- AGUIAR OB, SILVA TM. Características socioeconômicas, do trabalho e de saúde de nutricionistas de hospitais municipais do Rio de Janeiro. Mundo Saúde. 2017; 41(1): 57-67.

2- AKUTSU RC. Os nutricionistas brasileiros: perfil profissional e demográfico. Rev Nutr. 2008: 21(1); 7-19.

3- ALENCAR GA, et al. WhatsApp como ferramenta de apoio ao ensino. In: Anais dos Workshops do IV Congresso Brasileiro de Informática na Educação; 2015, out 26-30. Maceió - AL. CBIE; 2015.

4- COLONETTI T, et al. Perfil e Inserção do Profissional Nutricionista Atuante na Atenção Básica na Região Carbonífera de Santa Catarina. Inova Saúde. 2015; 4(2): 61-75.

5- CONSELHO FEDERAL DO NUTRICIONISTA. Resolução no 417. Dispões sobre procedimentos nutricionais para atuação dos nutricionistas e dá outras providências, 2008.

6- CONSELHO FEDERAL DE NUTRICIONISTAS. Inserção profissional dos nutricionistas no Brasil, 2006. Disponível em: http://www.cfn.org.br/novosite/pdf/pesquisa.pdf.

7- CONSELHO FEDERAL DE NUTRICIONISTAS. Perfil da atuação profissional do nutricionista no Brasil. 2006. Disponível em: http://www.cfn.org.br/eficiente/repositorio/Cartilhas/59.pdf.

8- FURLANETTO GS. O WhatsApp nas redações jornalísticas: um estudo exploratório. Rev Bras Inic Cien. 2017; 4(4): 184-198.

9- MELLO AL, et al. Perfil do nutricionista do programa nacional de alimentação escolar na região Nordeste do Brasil. Rev Nutr. 2012; 25(1):119-132.

10- QUADROS MRR, DIAS JS, MORO CMC. Análise das funções disponíveis nos softwares brasileiros de apoio à nutrição clínica. Curitiba: Nutroclínica, 2004.

11- OLIVEIRA EDS, et al. Experiência de uso do Whatsapp como Ambiente Virtual de Aprendizagem em um curso a distância. In: 3ํㅡㄹ Congresso Brasileiro de Informática na Educação; 2014, nov 03-06. Dourados - MS. Universidade Federal de Grande Dourados; 2014.

12- PEREIRA PC, et al. Ambientes virtuais e mídias de comunicação, abordando a explosão das mídias na sociedade da informação e seu impacto na aprendizagem - o uso do WhatsApp como plataforma de m-learning. Rev Mosaic. 2015; 06(1): 29-41.

13- RAIMAN L, et al. WhatsApp messenger as a tool to supplement medical education for medical students on clinical attachment. Med Educ. 2017; 17(7): 2-9.

14- RECINE E, et al. A formação em saúde pública nos cursos de graduação de nutrição no Brasil. Rev Nutr Camp. 2012; 25(1): 21-33.

15- ROCHA D, et al. "Whatsapp": de mensageiro instântaneo e chamada de voz em smartphones, para dispositivo de comunicação ubíqua dos gestores EAD da UFT/UAB no cerrado tocantinense. Rev Desafios. 2017; 4(2): 185-193.

16- RODRIGUES MK, et al. Condições de trabalho e perfil profissional dos nutricionistas egressos da Universidade Federal de Ouro Preto, Minas Gerais, entre 1994 e 2001. Ciência \& Saúde Coletiva. 2007; 12(4):1021-1031.

17- SOAR C, SILVA CAM. Perfil e carreira de egressos de Nutrição da Região do Vale do Paraíba-SP. Demetra. 2017; 12(4): 1013-1029.

18- SOUZA AG, et al. WhatsApp, inovação empreendedorismo. Rev Expr. 2014; 1(7): 1-19.

19- SOUZA JCN, et al. Formação, satisfação e perspectivas profissionais de egressos nutricionistas de uma universidade federal do nordeste brasileiro. Demetra. 2018; 13(1): 5-20.

20- SOUZA JLA, et al. Mídia social whatsapp: uma análise sobre as interações sociais. Rev Alterjor. 2015: 11(1); 1-35.

21- TEIXEIRA GA. WhatsApp como ferramenta estratégica de comunicação interna: um estudo de caso numa empresa do setor de bebidas em Picos-PI. Trabalho de conclusão de curso (Bacharelado em Administração) - Universidade Estadual do Piauí, Picos, Piauí, 2017; 54p. 